\title{
Impact of Depression on Hospitalization and Related Outcomes for Parkinson's Disease Patients: A Nationwide Inpatient Sample-Based Retrospective Study
}

Rikinkumar S. Patel ${ }^{1}$, Ramkrishna Makani ${ }^{2}$, Zeeshan Mansuri ${ }^{3}$, Upenkumar Patel ${ }^{4}$, Rupak Desai $^{5}$, Amit Chopra 6

1. Department of Global Public Health, Arcadia University, Philadelphia, USA 2. Child and Adolescent Psychiatry, Children's Hospital of Philadelphia 3. Psychiatry, Texas Tech University Health Sciences Center at Odessa/permian Basin 4. Public Health, National University 5. Research Fellow, Atlanta Veterans Affairs Medical Center, Decatur, Georgia 6. Psychiatry, Allegheny Health Network

$\square$ Corresponding author: Rikinkumar S. Patel, rpatel_09@arcadia.edu

Disclosures can be found in Additional Information at the end of the article

\section{Abstract}

\section{Background}

Major Depressive Disorder (MDD) is a common comorbidity that significantly affects the quality of life and disease outcomes in Parkinson's disease (PD) patients. No studies have been conducted to our knowledge to address the health care utilization and its outcomes in these patients. The aim of this study is to analyze and discern the differences in the hospitalization outcomes, comorbid conditions, and utilization of procedures in PD patients versus patients with comorbid MDD.

\section{Methods}

We used the Nationwide Inpatient Sample from the Healthcare Cost and Utilization Project from year's 2010-2014. We identified PD and MDD as a primary and secondary diagnosis respectively using validated International Classification of Diseases, 9th Revision, and Clinical Modification codes. Pearson's chi-square test and independent sample T-test were used for categorical data and continuous data, respectively. All statistical analysis was done by SPSS 22.0 in this study.

\section{Results}

Received 08/05/2017 Review began 08/22/2017 Review ended 08/28/2017 Published 09/03/2017

C) Copyright 2017

Patel et al. This is an open access article distributed under the terms of the Creative Commons Attribution License CC-BY 3.0., which permits unrestricted use, distribution, and reproduction in any medium, provided the original author and source are credited.
Extensive analysis was performed on 63,912 patients with PD and 1445 patients with PD having MDD. Patients with comorbid depression had three times greater chances of disposition to acute care hospital (3.1\% vs. 1.1\%, p < 0.001). Median length of hospitalization was higher in Parkinson's patients with depression (5.85 vs. 4.08 days; $\mathrm{p}<0.001$ ) though the median cost of hospitalization was low ( $\$ 31,039$ vs. $\$ 39,464 ; \mathrm{p}<0.001$ ). This could be because therapeutic procedures performed during the hospitalization were lower in Parkinson's patients with depression (0.53 vs. 0.89, p < 0.001). Utilization of Deep Brain Stimulation (DBS) was lower in Parkinson's patients with depression (9.4\% vs. 25.6\%, p < 0.001). In-hospital mortality was significantly higher in Parkinson's patients with depression (1.4\% vs. $1.1 \%$; $\mathrm{p}<0.001)$.

\section{Conclusion}

Our study establishes the negative impact of depression in PD with regards to hospitalization- 
related outcomes including the illness severity, comorbid conditions, risk of mortality, utilization of diagnostic and therapeutic procedures, the length of stay and disposition as compared to PD without depression.

Categories: Neurology, Psychiatry, Quality Improvement

Keywords: parkinson's disease, depression, parkinsonism, mdd, deep brain stimulation, dbs, comorbidities

\section{Introduction}

Parkinson's disease (PD) is a chronic, gradually progressive and fatal neurological disorder of the brain. The degeneration of Dopamine neurons in substantia nigra and deficiency of dopamine in the putamen (the regions involved in the regulation of movement) lead to cardinal clinical symptoms [1]. In the United States, the second most common neurodegenerative disease is PD with an estimated prevalence of $0.3 \%$ throughout the entire population [2]. The overall prevalence and global economic burden of PD in the world's top 10 densely populated nations were investigated. It is estimated that the PD occurrence rate will double by 2030 [3]. One million people in America are affected by the PD and there are over 60,000 new cases reported every year [4-5].

Since there are no explicit tests to diagnose PD, it remains a clinical diagnosis based on the patient's report and the combination of exhibited symptoms. The primary motor symptoms of PD include tremor, rigidity, akinesia or bradykinesia, and postural instability. The non-motor symptoms of PD include sleep disorders and psychiatric disorders including depression and anxiety. These non-motor symptoms have a huge influence that can negatively impact the health-related life quality of PD patients [6]. The severity of such symptoms indicates the progression of the disease, which results in higher costs of medical treatment for hospitalization and care coordination. Among the psychiatric disorders comorbid with PD, depression has the highest prevalence, followed by anxiety disorders, sleep disorders, and others [7]. Diagnosis of patients with PD is 1.89-4.26 times likely to get depression [8]. The severity of depression appears to correlate with disability and reduced quality of life in PD patients [9]. Depression seems to be underdiagnosed and undertreated in PD [7], even though adequate diagnosis and treatment can change the direction of the illness positively. Reduction of quality of life by depression in PD is independent of motor deficits. Therefore, depression should be treated independently of motor symptoms in patients with PD. Aspects of quality of life have been emphasized in diagnosis and treatment of PD and there appears to be relevant response criteria and goals in the pharmacological and non-pharmacological treatment of PD [10]. The impact of depression in PD patients ranges from mild manifested as an unwillingness to cooperate to a complete withdrawal and social isolation [11].

Previously studies were done to measure prevalence, neurobiology, and impact on healthrelated quality of life in PD patients with depression [12-14]. However, no previous studies were conducted to the best of our knowledge to address the health care utilization and hospitalization outcomes in PD patients with depression. Studies in the past evaluated medical and psychiatric comorbidities present in PD patients [15-17]. This study focussed on comorbidities that were present in PD with major depressive disorder (MDD) patients and compared its prevalence with PD patients without depression.

The main aims of this study are to analyze and discern the differences in the hospitalization outcomes, comorbid conditions and utilization of therapeutic and diagnostic procedures in PD patients versus PD patients with MDD. The results obtained from this study can be used effectively to assess the burden of care of MDD in PD and to develop strategies, and clinical care models to recognize and treat MDD to improve the quality of life of the patients by 


\section{Materials And Methods}

\section{Data source}

A retrospective analysis was performed using the Healthcare Cost and Utilization Project's (HCUP) Nationwide Inpatient Sample (NIS) data from the years 2010 to 2014. The Agency for Healthcare Research and Quality (AHRQ) sponsors the HCUP databases that are specifically designed to determine and identify patterns in hospital utilization and cost across the United States. The HCUP-NIS database is the largest inpatient database available in the United States, which represents a sample of non-federal United States community hospitals. In 2010, there was an increase of 1,051 hospitals to 4,411 hospitals from 45 states in the United States that participated in NIS projects. More than seven million records of hospital stays were reported by these hospitals each year. A sample estimate of over $95 \%$ of discharges from the hospitals participated in NIS. We weighted the estimated samples to minimize the margin of error and to reflect all 50 states across the United States. The large sample size of the database enabled us to analyze rare conditions, uncommon treatments, and special patient populations. To protect the privacy of individual patients, physicians, and hospitals, the state and hospital identifiers are de-identified. There are many clinical and non-clinical hospitalization data elements recorded in the HCUP NIS database. Sample non-clinical information is patient's demographic data, hospital characteristics (such as region and location), and total charges. Sample clinical-related information includes principal and secondary diagnosis, procedure codes (includes both ICD-9 and CCS codes), discharge status and the length of stay [18].

\section{Variables of interest}

Based on the ICD-9-CM diagnosis codes, we identified the individuals with a primary diagnosis of PD at the time of admission. Then, based on the ICD-9-CM diagnosis codes, the people with a primary diagnosis of PD and secondary diagnosis as MDD patients at the time of admission had been identified as the comparison group. In HCUP databases, more than 14,000 ICD-9-CM diagnosis codes and 3,900 procedure codes had been mentioned which were further classified and clustered into a lesser number of clinically appropriate categories by the AHRQ's Clinical Classification Software (CCS). This enabled the database to capture a large population of relatively similar conditions into a single group by making the information more useful for performing statistical analyses and developing reports. PD was identified using diagnosis code 332 and MDD was identified using diagnosis codes 296.2 and 296.3. To measure the differences in hospitalization outcomes in PD patients versus PD with MDD patients, the outcome variables of interest included the severity of illness that measures the loss of body functions, the risk of mortality that measures the likelihood of dying, disposition of patient and in-hospital mortality. In the NIS, we defined death as in-hospital mortality, and in this paper, it is reported as all-cause. We calculated the length-of-stay as the number of nights the patient remained in the hospital for a particular discharge. Length-of-stay in this analysis was all-cause. Total charges of hospitalization do not include professional fees and non-covered charges. If the source provided total charges with professional fees, then the professional fees were removed from the charge during HCUP processing. For the analysis, among the predictor variables, transfers out of the current hospital setting, a total number of ICD-9 CM procedures, Primary ICD-9 Procedures, and duration of the hospital stays were considered to be important. The primary and secondary diagnoses had been identified at the date of admission whereas we recorded comorbidities throughout the entire hospital stay. By a common definition, comorbidities were considered coexisting conditions with PD and PD with MDD, the index disorders under study. AHRQ comorbidity software was used to generate binary variables that identified seven comorbidities in discharge records using ICD-9-CM codes [19]. ICD-9 CM codes used for alcohol abuse are 291.0-291.3, 291.5, 291.8, 291.81, 281.82, 291.89, 291.9, 303.00303.93 and 305.00-305.03; drug abuse are 292.0, 292.82-292.89, 292.9, 304.00-304.93, 305.20- 
305.93 and 648.30-648.34; psychosis are 295.00-298.9, 299.10 and 299.11; delirium are 290.11, 290.3, 290.41, 292.81, 293, 293.1, 292.11 and 292.12; and dementia are 290.0, 290.10-290.13, 290.20, 290.21, 290.3, 290.40-290.43, 294.0, 294.1, 331.0, 331.11, 331.19, 331.82, 331.89, 332.0 and 333.0.

\section{Approaches}

A retrospective analysis was performed over the HCUP-NIS database focusing on the determination of the hospital outcomes for PD and PD with MDD patients. Descriptive statistics were used to summarize the results. The mean and standard deviations were used to explain the continuous variables. Pearson's chi-square test and independent sample T-test were used for categorical data and continuous data, respectively. On the other hand, the categorical variables were shown in percentage values. We used discharge weight which is given in NIS database to obtain national represent inpatient data. A p-value $<0.05$ was used as a reference to determine the statistical significance test result. All statistical analysis was done by SPSS 22 in this study [20]. Our study database does not contain patient identification. Thus, we were not required to take Institution Review Board (IRB) permission for this study.

\section{Results}

Extensive analysis was performed on 63,912 patients with PD and 1,445 patients with PD having MDD according to the hospital records. The preliminary results are presented below.

\section{Demographics}

Age Distribution

The patients were distributed according to their age group as shown in Table 1 and Figure 1 . The result indicates that a total of $62.8 \%$ of PD patients and $63.4 \%$ of PD with MDD patients were in the range of 65 to 84 years age group, comprising the most common age of presentation of PD and PD with MDD (p-value < 0.001). PD with MDD was less common in above 85 years age group which comprised of $8.5 \%$ of total PD with MDD patients in comparison to $12.5 \%$ of total PD patients ( $p$-value $<0.001$ ). The mean age of PD patients at the time of hospitalization was 71.7 years $($ S.D. $=11.296$, $\mathrm{p}$-value $=0.262)$ and 69.5 years $($ S.D. $=11.366, p$-value $=0.262)$ for PD with MDD patients. 


\section{Cureus}

\begin{tabular}{|c|c|c|c|}
\hline Age (in years) & PD & PD with MDD & p-value \\
\hline Mean Age \pm S.D. & $71.7 \pm 11.296$ & $69.5 \pm 11.366$ & 0.262 \\
\hline $1-17$ & $0.1 \%$ & $0 \%$ & $<0.001$ \\
\hline $18-44$ & $1.4 \%$ & $2.7 \%$ & $<0.001$ \\
\hline $45-64$ & $23.2 \%$ & $25.4 \%$ & $<0.001$ \\
\hline $65-84$ & $62.8 \%$ & $63.4 \%$ & $<0.001$ \\
\hline$>85$ & $12.5 \%$ & $8.5 \%$ & $<0.001$ \\
\hline
\end{tabular}

TABLE 1: Distribution of Parkinson's disease and Parkinson's disease with major depressive disorder patients by the age group.

Significant $p$-values $\leq 0.05$ at $95 \%$ confidence interval.

PD: Parkinson's disease; MDD: Major depressive disorder.

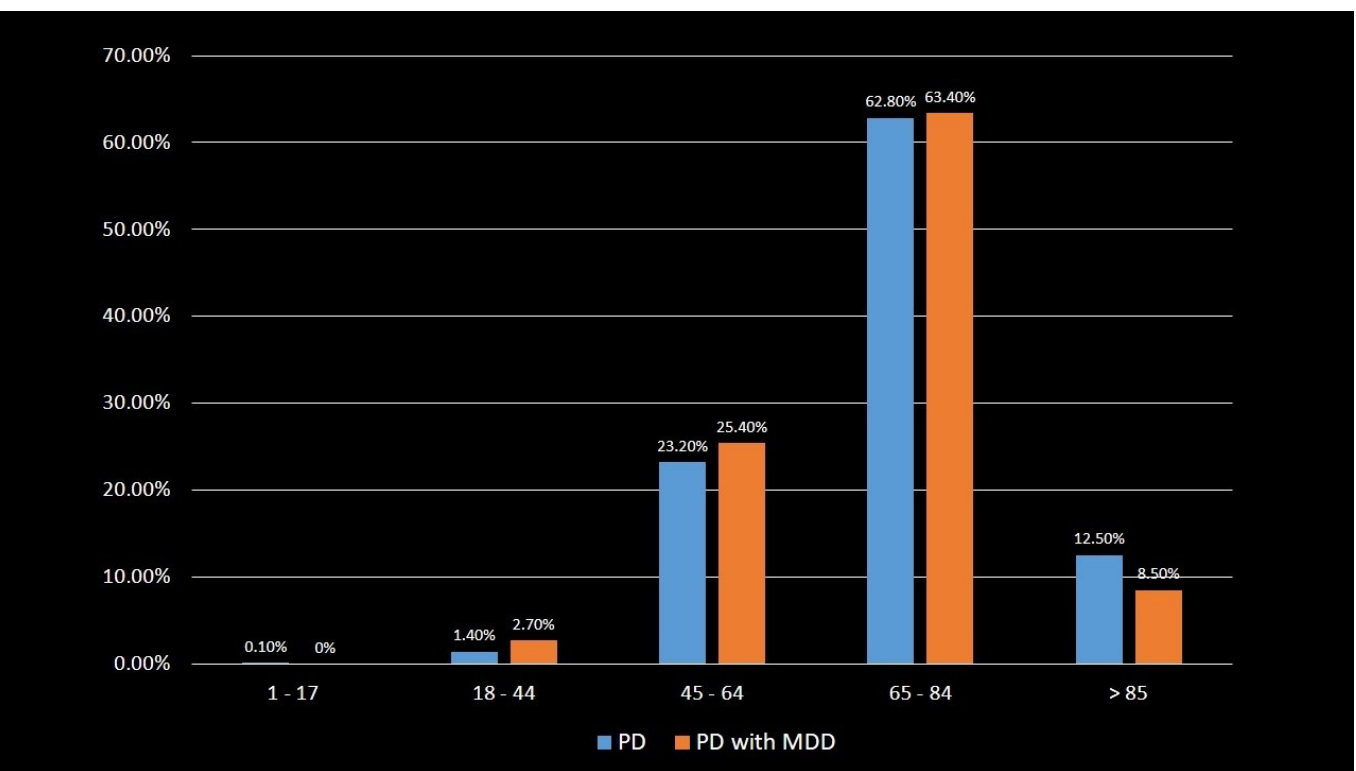

FIGURE 1: Distribution of Parkinson's disease and Parkinson's disease with major depressive disorder patients by the age group.

PD: Parkinson's disease; MDD: Major depressive disorder.

\section{Sex Distribution}

The patients were distributed according to their sex as shown in Table 2 and Figure 2. PD was found in the greater proportion of males (62.7\%), whereas PD with MDD was in the higher 


\section{Cureus}

proportion of females $(52.2 \%)(\mathrm{p}$-value $<0.001)$.

\begin{tabular}{|c|c|c|c|}
\hline Sex & PD & PD with MDD & p-value \\
\hline Male & $62.7 \%$ & $37.3 \%$ & $<0.001$ \\
\hline Female & $47.8 \%$ & $52.2 \%$ & $<0.001$ \\
\hline
\end{tabular}

\section{TABLE 2: Distribution of Parkinson's disease and Parkinson's disease with major} depressive disorder patients by the sex.

Significant $p$-values $\leq 0.05$ at $95 \%$ confidence interval.

PD: Parkinson's disease; MDD: Major depressive disorder.

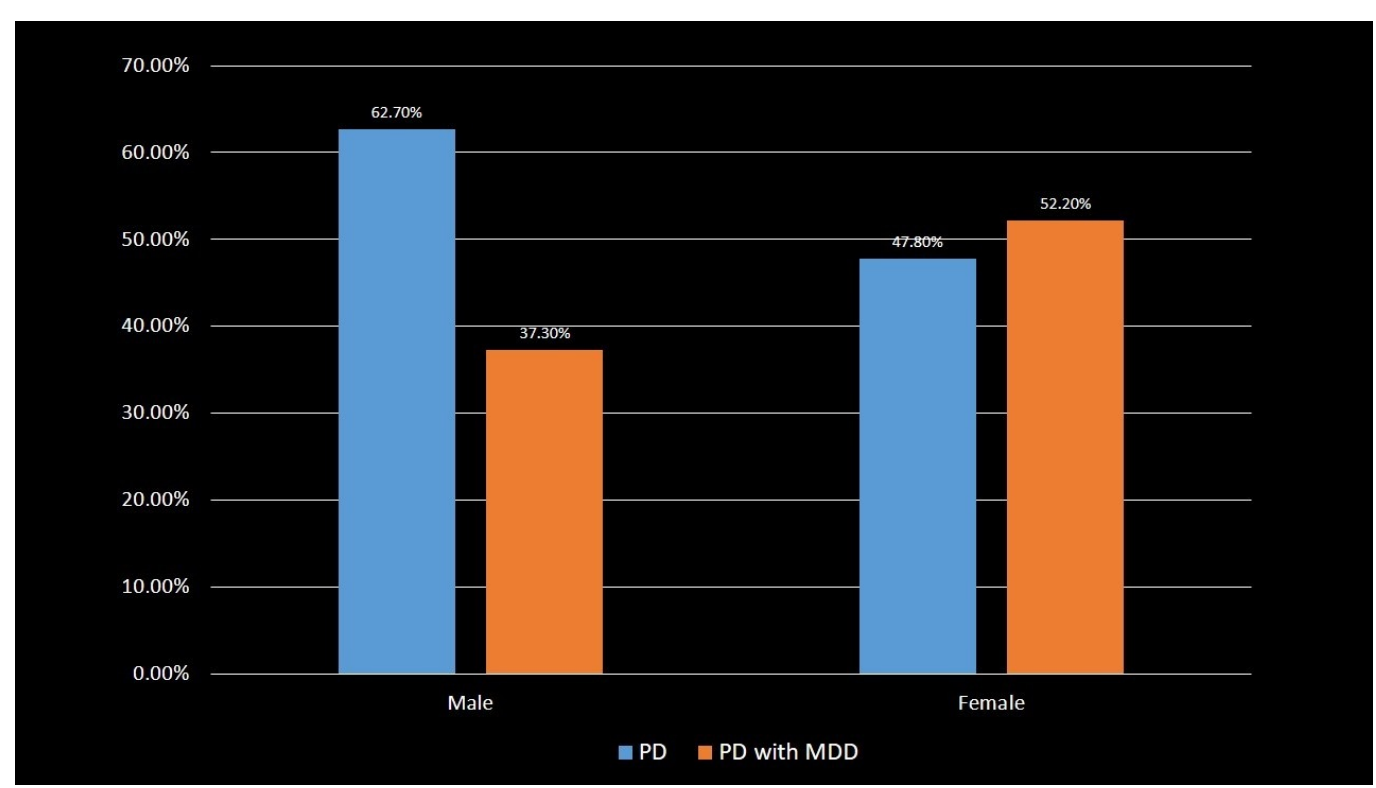

FIGURE 2: Distribution of Parkinson's disease and Parkinson's disease with major depressive disorder patients by the sex.

PD: Parkinson's disease; MDD: Major depressive disorder.

Race Distribution

The patients were distributed according to their race as shown in Table 3 and Figure 3. PD was discovered in $80.3 \%$ of Whites and PD with MDD was found in $76.2 \%$ of Whites. The White race is an important risk factor for PD and PD with MDD. A greater proportion of PD with MDD was found in Hispanics (11.5\%) and Asian or Pacific Islanders (4.2\%) as compared to PD patients (6.7\% and $2.3 \%$, respectively) (p-value $<0.001)$. 


\section{Cureus}

\begin{tabular}{|c|c|c|c|}
\hline Race & PD & PD with MDD & p-value \\
\hline White & $80.3 \%$ & $76.2 \%$ & $<0.001$ \\
\hline Black & $7 \%$ & $5.4 \%$ & $<0.001$ \\
\hline Hispanic & $6.7 \%$ & $11.5 \%$ & $<0.001$ \\
\hline Asian or Pacific Islander & $2.3 \%$ & $4.2 \%$ & $<0.001$ \\
\hline Native American & $0.5 \%$ & $0.8 \%$ & $<0.001$ \\
\hline Other & $3.2 \%$ & $1.9 \%$ & $<0.001$ \\
\hline
\end{tabular}

\section{TABLE 3: Distribution of Parkinson's disease and Parkinson's disease with major}

depressive disorder patients by the race.

Significant $p$-values $\leq 0.05$ at $95 \%$ confidence interval.

PD: Parkinson's disease; MDD: Major depressive disorder.

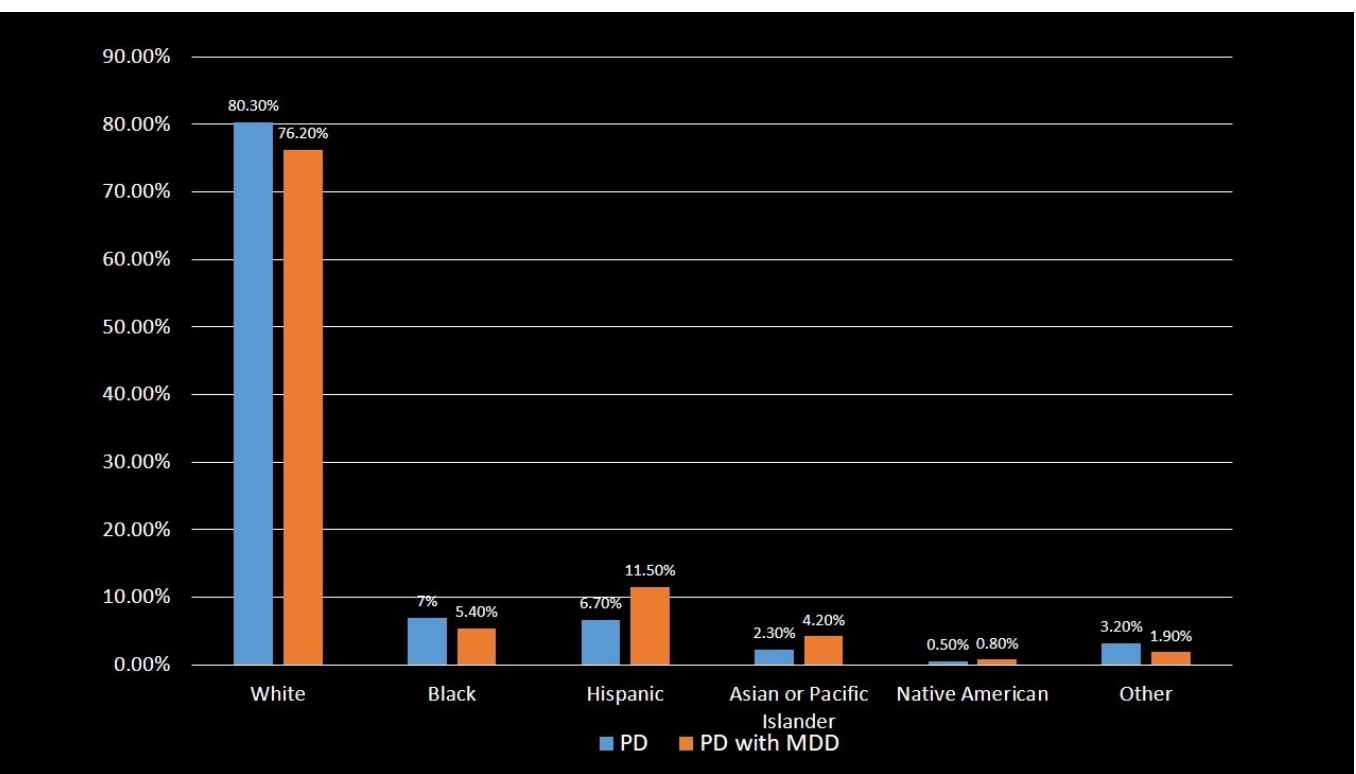

FIGURE 3: Distribution of Parkinson's disease and Parkinson's disease with major depressive disorder patients by the race.

PD: Parkinson's disease; MDD: Major depressive disorder.

Geographic Region Distribution

The patients were distributed according to their geographic region as shown in Table 4 and Figure 4. The highest number of PD patients was found in Midwest (28.4\%) and Southern (28.4\%) regions of the United States, while the highest number of PD with MDD patients was 


\section{Cureus}

found in Southern (34.6\%) region of United States ( $\mathrm{p}$-value < 0.001). Prevalence of PD was lowest in Northeast region (20\%), and PD with MDD was lowest in the Western region (18.2\%) (p-value $<0.001)$.

\begin{tabular}{|c|c|c|c|}
\hline Region & PD & PD with MDD & p-value \\
\hline Northeast & $20 \%$ & $23.7 \%$ & $<0.001$ \\
\hline Midwest & $28.4 \%$ & $23.5 \%$ & $<0.001$ \\
\hline South & $28.4 \%$ & $34.6 \%$ & $<0.001$ \\
\hline West & $23.2 \%$ & $18.2 \%$ & $<0.001$ \\
\hline
\end{tabular}

\section{TABLE 4: Distribution of Parkinson's disease and Parkinson's disease with major} depressive disorder patients by the geographic region.

Significant $p$-values $\leq 0.05$ at $95 \%$ confidence interval.

PD: Parkinson's disease; MDD: Major depressive disorder.

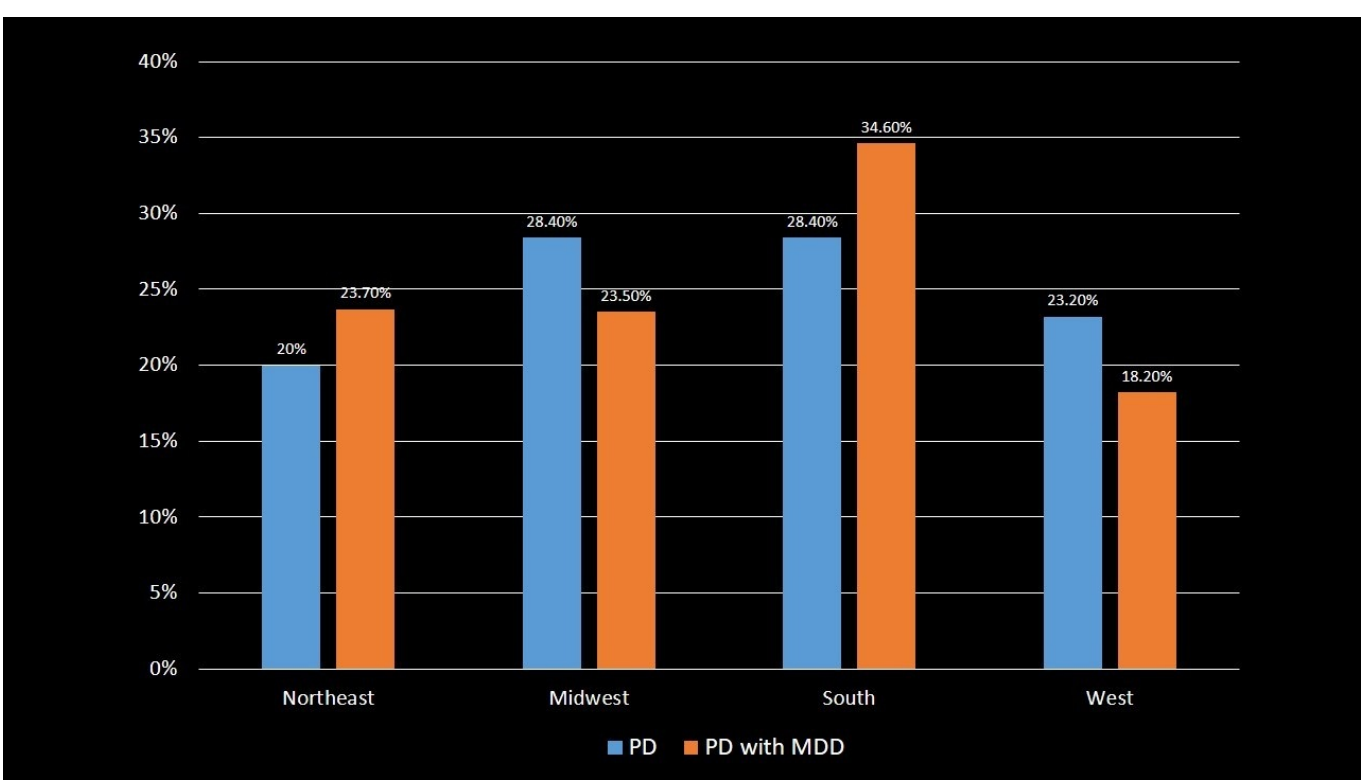

FIGURE 4: Distribution of Parkinson's disease and Parkinson's disease with major depressive disorder patients by the geographic region.

PD: Parkinson's disease; MDD: Major depressive disorder.

\section{Disposition/transfer of patient}

The patients were distributed according to transfer/disposition as shown in Table 5 and Figure 


\section{Cureus}

5. A major proportion of PD with MDD patients (50.6\%) and PD patients (42.1\%) was transferred to skilled nursing facility (SNF), intermediate nursing facility (INF) or another type of facility. PD with MDD patients had three times greater chances of disposition to short-term or acute care hospital, as 3.1\% PD with MDD patients were transferred to such facilities in comparison to $1.1 \%$ PD patients ( $\mathrm{p}$-value $<0.001$ ). This indicates that greater numbers of PD with MDD patients needed a specialized care in comparison to PD patients.

\begin{tabular}{|c|c|c|c|}
\hline Disposition of patient & PD & PD with MDD & p-value \\
\hline Not a transfer/routine & $56.8 \%$ & $46.4 \%$ & $<0.001$ \\
\hline To acute care hospitals & $1.1 \%$ & $3.1 \%$ & $<0.001$ \\
\hline Other (SNF, INF, another facility) & $42.1 \%$ & $50.6 \%$ & $<0.001$ \\
\hline
\end{tabular}

TABLE 5: Distribution of Parkinson's disease and Parkinson's disease with major depressive disorder patients by the transfer/disposition.

Significant $p$-values $\leq 0.05$ at $95 \%$ confidence interval.

PD: Parkinson's disease; MDD: Major depressive disorder; SNF: Skilled nursing facility; INF: Intermediate nursing facility.

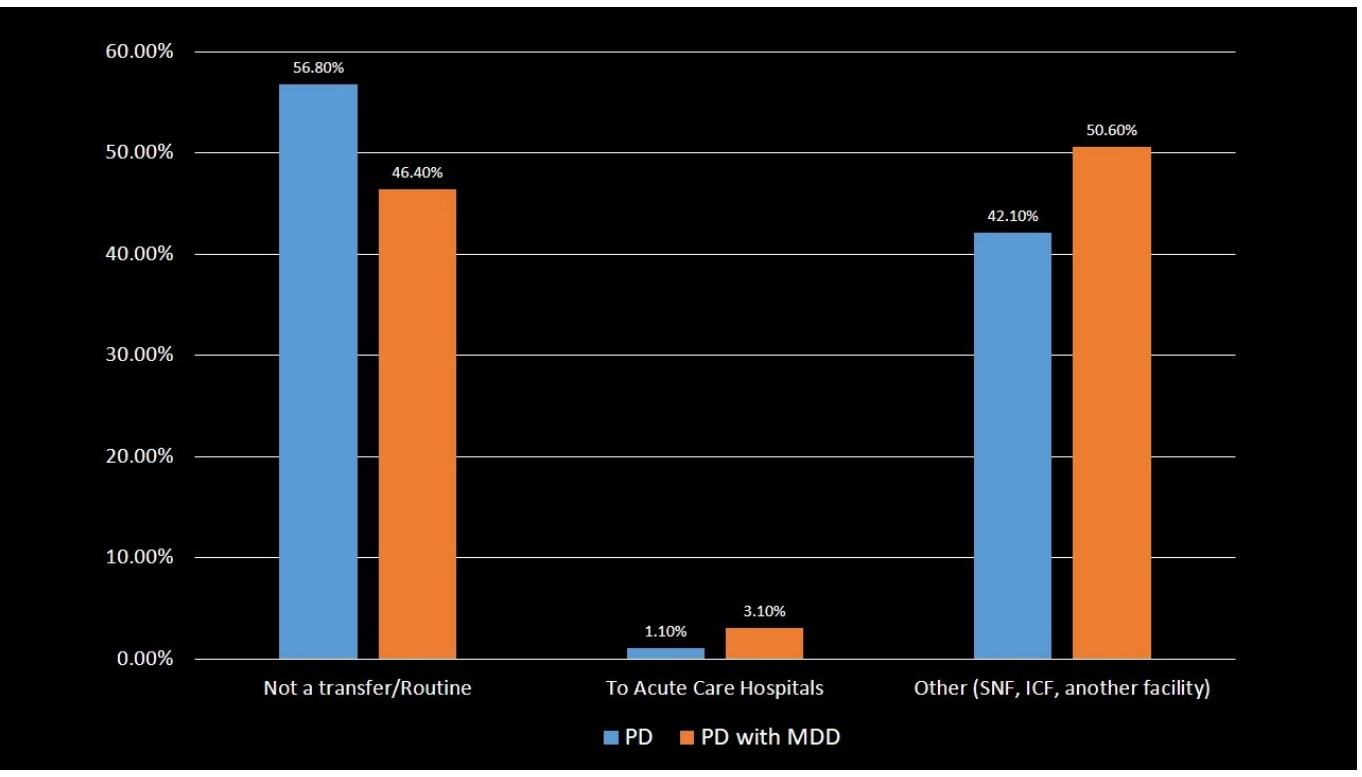

FIGURE 5: Distribution of Parkinson's disease and Parkinson's disease with major depressive disorder patients by the transfer/disposition.

PD: Parkinson's disease; MDD: Major depressive disorder; SNF: Skilled nursing facility; INF: Intermediate nursing facility.

\section{Risk of mortality}




\section{Cureus}

About $17 \%$ and $2.3 \%$ of PD with MDD patients had a major and extreme probability of dying respectively, which was higher as compared to $13.2 \%$ and $1.9 \%$ of PD patients with the risk of major and extreme probability of dying, respectively (p-value $<0.001$ ). This indicates that PD with MDD patients are at higher risk of in-hospital mortality as compared to PD patients. The patients were distributed according to the risk of mortality in Table 6 and Figure 6.

\begin{tabular}{|c|c|c|c|}
\hline Risk of mortality & PD & PD with MDD & p-value \\
\hline Minor & $47.2 \%$ & $41.4 \%$ & $<0.001$ \\
\hline Moderate & $37.6 \%$ & $39.4 \%$ & $<0.001$ \\
\hline Major & $13.2 \%$ & $1 / \%$ & $<0.001$ \\
\hline Extreme & $1.9 \%$ & $2.3 \%$ & $<0.001$ \\
\hline
\end{tabular}

TABLE 6: Risk of mortality in Parkinson's disease and Parkinson's disease with major depressive disorder patients.

Significant $p$-values $\leq 0.05$ at $95 \%$ confidence interval.

PD: Parkinson's disease; MDD: Major depressive disorder.

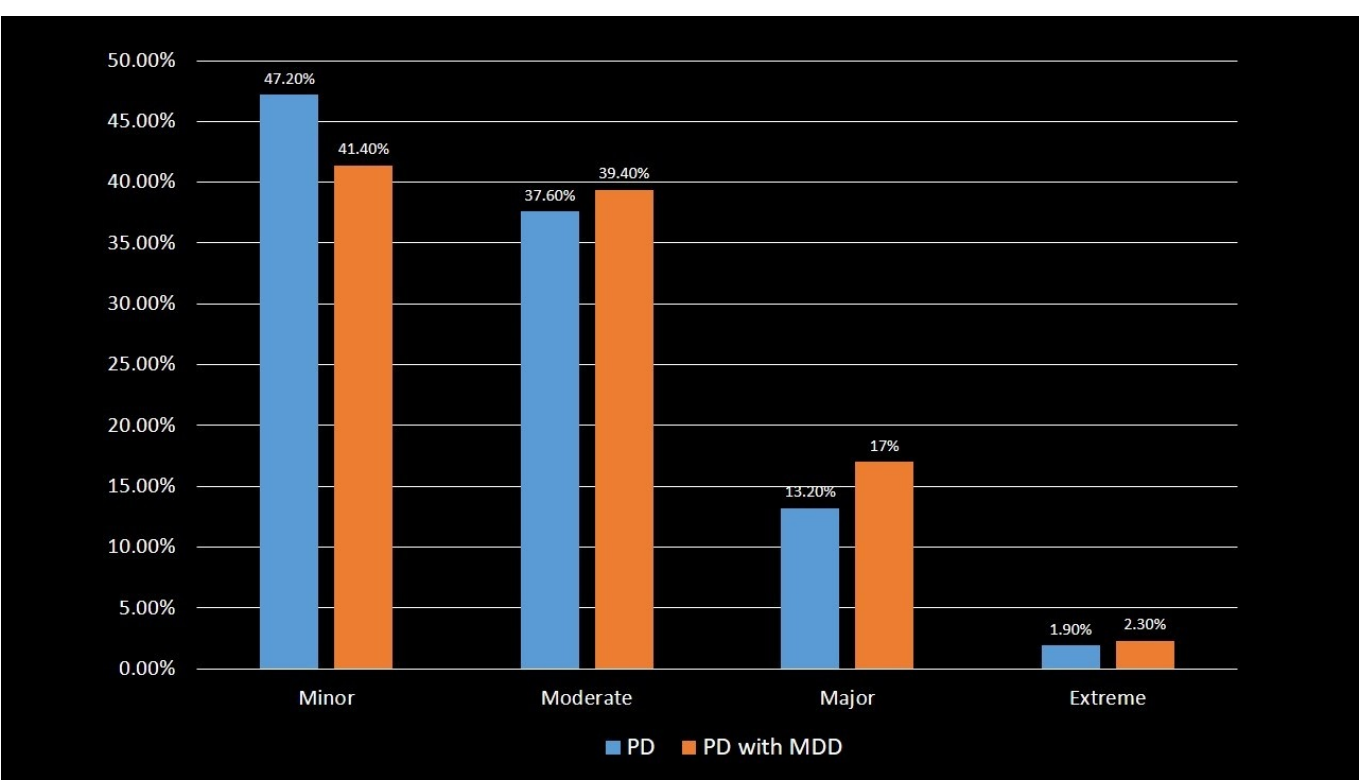

FIGURE 6: Risk of mortality in Parkinson's disease and Parkinson's disease with major depressive disorder patients.

PD: Parkinson's disease; MDD: Major depressive disorder.

\section{Severity of illness}

About 33.3\% and 4.1\% of PD with MDD patients had a risk of major and extreme loss of 


\section{Cureus}

function respectively, which was higher as compared to $19.6 \%$ and $2.4 \%$ of PD patients with a risk of major and extreme loss of function respectively ( $\mathrm{p}$-value $<0.001$ ). This indicates that PD with MDD patients have about twice greater risk of major or extreme loss of function as compared to PD patients. The patients were distributed according to the severity of illness in Table 7 and Figure 7.

\begin{tabular}{|c|c|c|c|}
\hline Severity of illness & PD & PD with MDD & p-value \\
\hline Minor & $33.5 \%$ & $11.8 \%$ & $<0.001$ \\
\hline Moderate & $44.5 \%$ & $50.8 \%$ & $<0.001$ \\
\hline Major & $19.6 \%$ & $33.3 \%$ & $<0.001$ \\
\hline Extreme & $2.4 \%$ & $4.1 \%$ & $<0.001$ \\
\hline
\end{tabular}

\section{TABLE 7: Severity of illness in Parkinson's disease and Parkinson's disease with} major depressive disorder patients.

Significant $p$-values $\leq 0.05$ at $95 \%$ confidence interval.

PD: Parkinson's disease; MDD: Major depressive disorder.

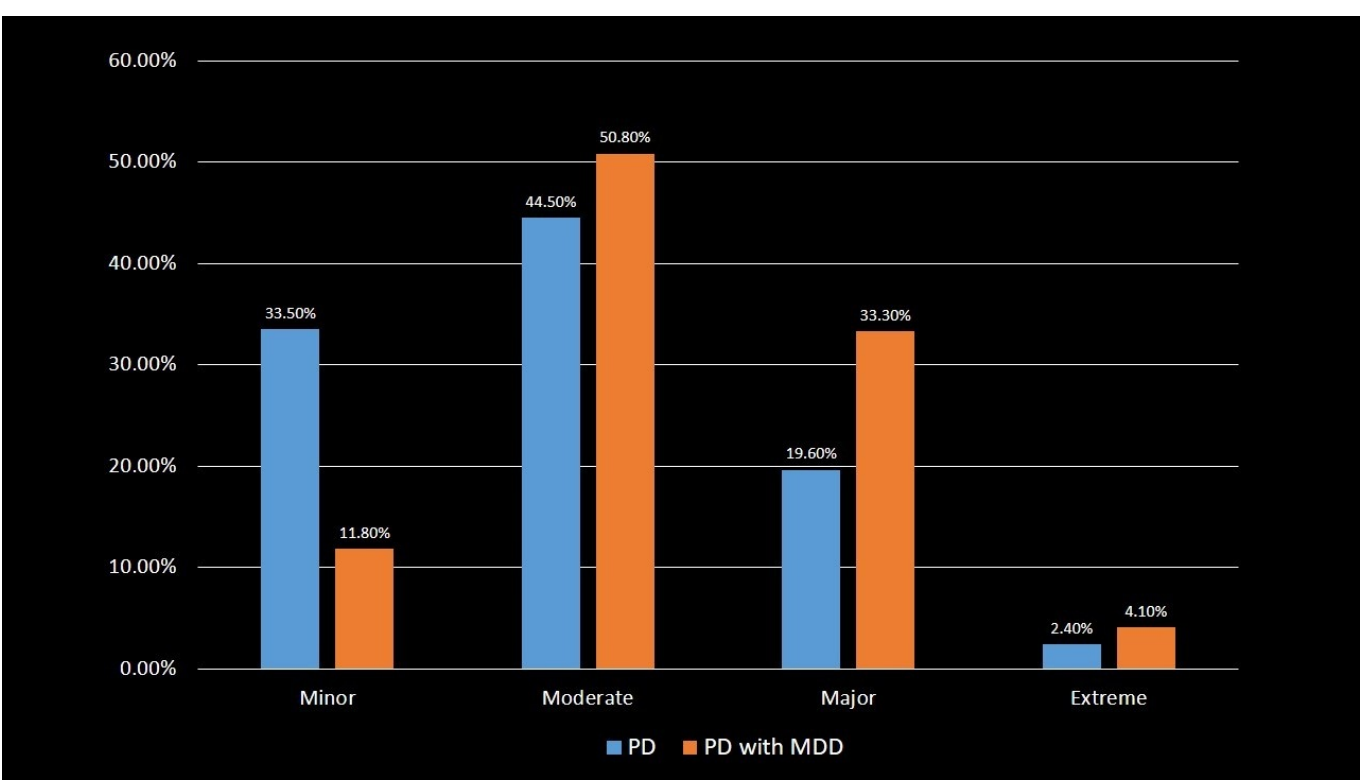

FIGURE 7: Severity of illness in Parkinson's disease and Parkinson's disease with major depressive disorder patients.

PD: Parkinson's disease; MDD: Major depressive disorder.

\section{Length of stay}

The average duration of stay for PD with MDD patients was 5.85 days (S.D. $=7.323$; p-value < 
0.001), which was higher as compared to PD patients who had the average length of stay for 4.08 days (S.D. $=8.511 ;$ p-value $<0.001$ ).

\section{Total charges of hospitalization}

Average total charges of hospitalization of PD with MDD patients were $\$ 31,038.99$ (S.D. = 32537.986; p-value < 0.001) which was lower as compared to the $\$ 39,463.89$ (S.D. $=43656.466$; p-value $<0.001$ ) average total charges for hospitalization of PD patients. PD with MDD patients had lower hospitalization expense as compared to PD patients despite having a longer duration of stay in the hospital. This could be due to less number of therapeutic procedures utilized in PD with MDD patients which is discussed in later part of this section.

\section{In-hospital mortality}

The risk of in-hospital mortality was higher in PD with MDD patients as compared to PD patients without depression. A total of 1.4\% PD with MDD patients died in the hospital at the time of recent admission, which raised the in-hospital mortality of these patients as compared to $1.1 \%$ PD patients who passed away in the hospital ( $\mathrm{p}$-value $<0.001$ ).

\section{Procedures}

Average number of procedures performed during the hospitalization was higher in PD patients, i.e., 0.89 (S.D. $=1.309$, p-value < 0.001) compared to 0.53 procedures in PD with MDD patients (S.D. $=0.977$, p-value $<0.001$ ). A total of 29,343 (45.9\%) PD patients and 437 (30.2\%) PD with MDD patients underwent therapeutic or diagnostic procedures during the hospitalization. The most common procedure performed was Therapeutic Nervous system procedure, i.e., 59.5\% in PD patients, but lower in PD with MDD patients (29.9\%). Diagnostic procedures like computed tomography (CT)-scan Head, magnetic resonance imaging (MRI) scan and diagnostic spinal tap were performed more in PD with MDD patients (5.5\%, 6.6\% and 3.4\%, respectively) compared to $\mathrm{PD}$ patients $(4.2 \%, 2.8 \%$ and $2.5 \%$, respectively) ( $\mathrm{p}$-value $<0.001)$. Invasive procedures like Gastrotomy (temporary or permanent placement) were performed 1.5 times greater in PD with MDD patients as compared to PD patients ( $p$-value $<0.001$ ). The patients were distributed according to the utilization of procedures in Table 8 and Figure 8 . 


\section{Cureus}

\begin{tabular}{|c|c|c|c|}
\hline Procedure & PD & PD with MDD & p-value \\
\hline Therapeutic nervous system procedure & $59.5 \%$ & $29.9 \%$ & $<0.001$ \\
\hline Diagnostic spinal tap & $2.5 \%$ & $3.4 \%$ & $<0.001$ \\
\hline Gastrotomy & $2.8 \%$ & $4.6 \%$ & $<0.001$ \\
\hline CT-scan & $4.2 \%$ & $5.5 \%$ & $<0.001$ \\
\hline MRI-scan & $2.8 \%$ & $6.6 \%$ & $<0.001$ \\
\hline Other procedures & $28.2 \%$ & $50 \%$ & $<0.001$ \\
\hline
\end{tabular}

TABLE 8: Procedures in Parkinson's disease and Parkinson's disease with major depressive disorder patients.

Significant $p$-values $\leq 0.05$ at $95 \%$ confidence interval.

PD: Parkinson's disease; MDD: Major depressive disorder; CT: Computed tomography; MRI: Magnetic resonance imaging.

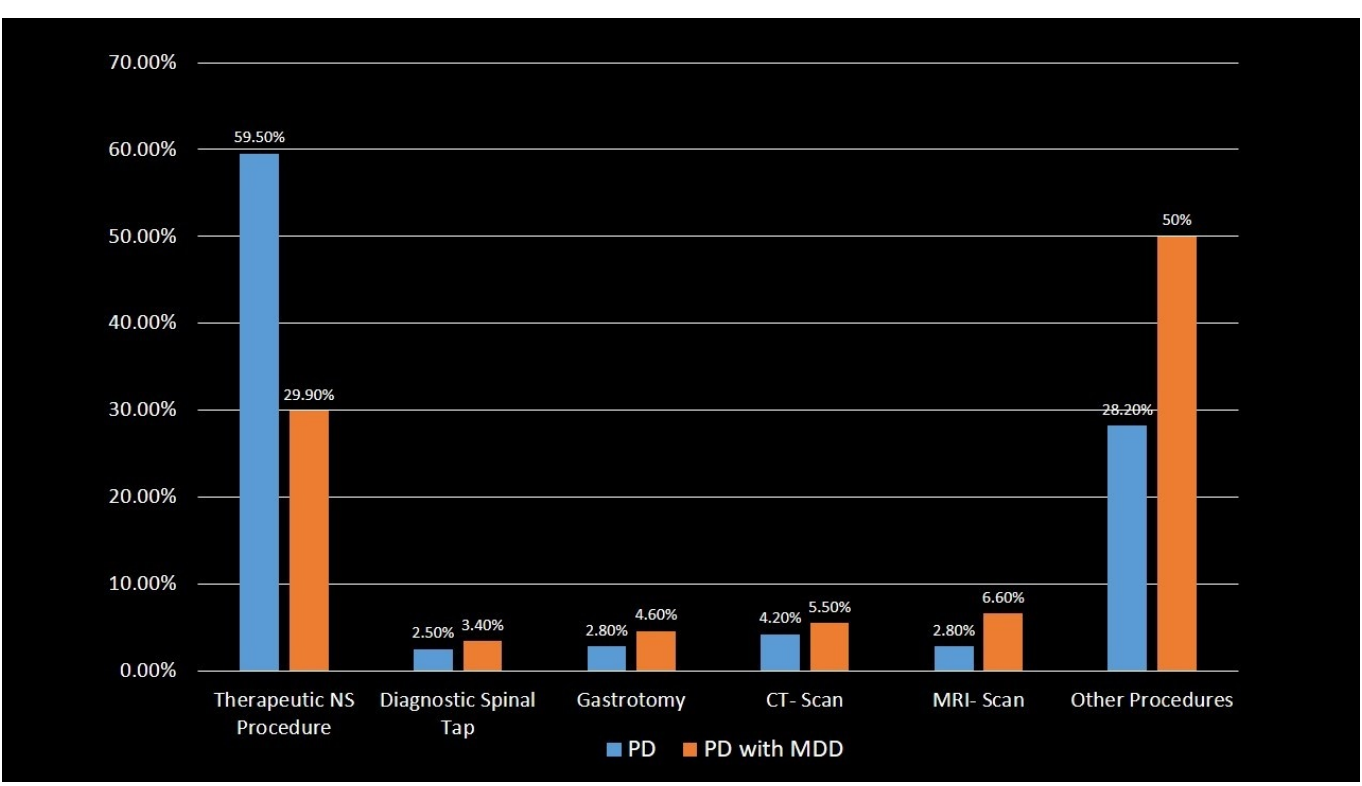

FIGURE 8: Procedures in Parkinson's disease and Parkinson's disease with major depressive disorder patients.

PD: Parkinson's disease; MDD: Major depressive disorder; NS: Nervous system; CT: Computed tomography; MRI: Magnetic resonance imaging.

The most important therapeutic procedure used during hospitalization is deep brain stimulation (DBS) in which there were 136, i.e., 9.4\% of total PD with MDD patients, which is very low as compared to the use of DBS in 16,368, i.e., 25.6\% of PD patients (p-value < 0.001 ). On the contrary, only 10 (0.7\%) PD with MDD patients and $396(0.6 \%)$ PD patients were treated 


\section{Cureus}

with stereotactic radiosurgery (SRS) during the hospitalization, though this result was not statistically significant ( $\mathrm{p}$-value $=0.728$ ). The patients were distributed according to the utilization of DBS and SRS procedures in Table 9 and Figure 9.

\begin{tabular}{|c|c|c|c|}
\hline Procedure & PD & PD with MDD & p-value \\
\hline DBS & $25.6 \%$ & $9.4 \%$ & $<0.001$ \\
\hline SRS & $0.6 \%$ & $0.7 \%$ & 0.728 \\
\hline
\end{tabular}

TABLE 9: Utilization of deep brain stimulation and stereotactic radiosurgery in Parkinson's disease and Parkinson's disease with major depressive disorder patients.

Significant $p$-values $\leq 0.05$ at $95 \%$ confidence interval.

PD: Parkinson's disease; MDD: Major depressive disorder; DBS: Deep brain stimulation; SRS: Stereotactic radiosurgery.

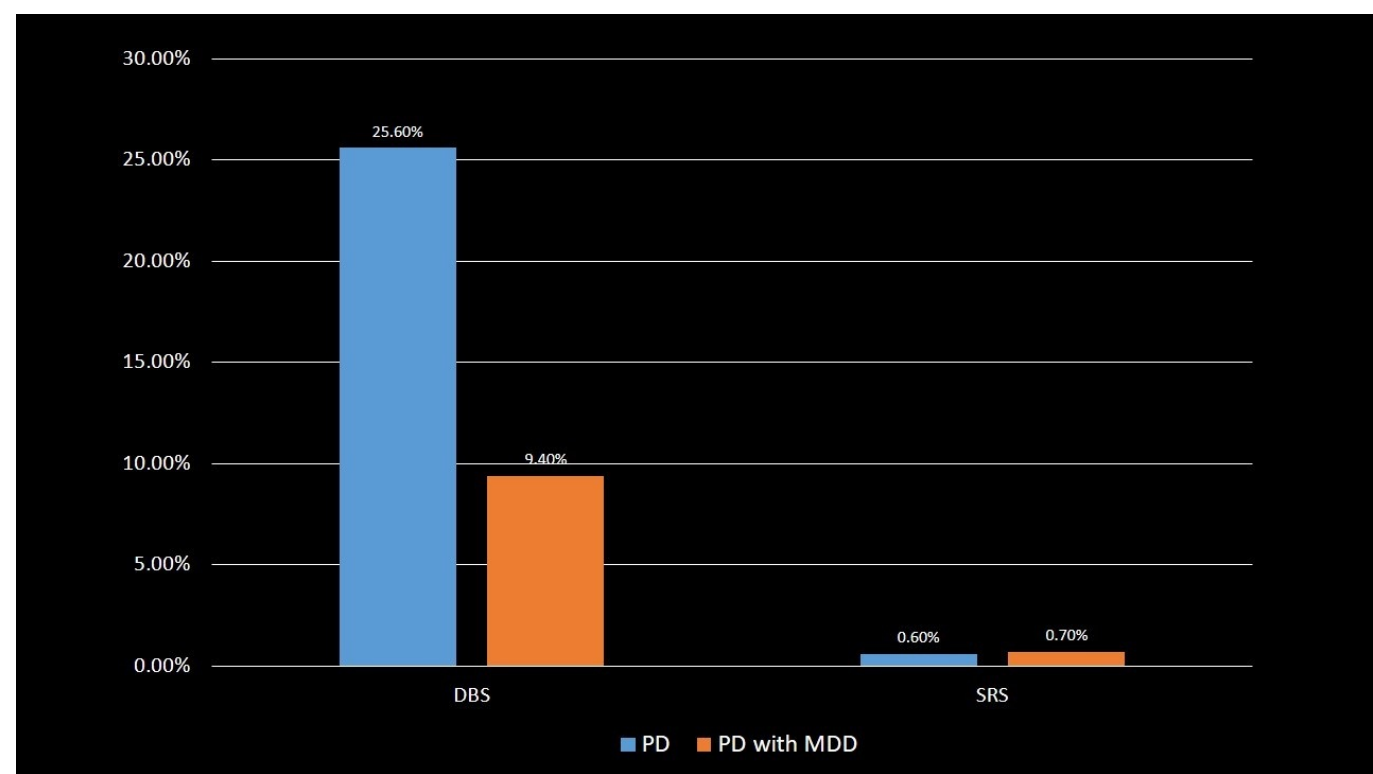

FIGURE 9: Utilization of deep brain stimulation and stereotactic radiosurgery in Parkinson's disease and Parkinson's disease with major depressive disorder patients.

PD: Parkinson's disease; MDD: Major depressive disorder; DBS: Deep brain stimulation; SRS: Stereotactic radiosurgery.

\section{Comorbidities}

Comorbidities present in PD with MDD patients are alcohol abuse (3.7\%), psychosis (100\%) and drug abuse (5\%) compared to $1.4 \%, 0 \%$, and $0.8 \%$ respectively in $\mathrm{PD}$ patients ( $\mathrm{p}$-value $<0.001$ ). Thus, psychosis was noted to be highly prevalent comorbidity in PD with MDD patients. 
Dementia was seen in $10.2 \%$ of PD and $11.5 \%$ of PD with MDD patients ( $p$-value $=0.059$ ). Delirium comorbidity was not present in PD patients but was seen in $0.3 \%$ of PD with MDD patients (p-value $<0.001$ ).

\section{Discussion}

This analysis of population-based hospital data from patients with PD and PD with MDD reveals the impact of depression on the hospitalization and related outcomes in PD. Prevalence rates of depressive syndromes in PD that are reported in the literature vary widely, ranging from 2.7\% to more than 90\% [21-22]. Possible reasons for this variation include the nature of the population studied, the way the diagnosis is established, the types of depressive disorders included in the study, and the statistical measures used. Our study had analyzed NIS data with hospitalization from 2010 to 2014 and over a five-year period, 2.26\% of patients with PD had a diagnosis of MDD. Patients with younger age at the onset appear to have higher rates of depression than those with later age at the onset [23]. Our study results supported the earlier observations, as the higher proportion of PD with MDD patients were present compared to PD patients in 45-64 years and 65-84 years age group as compared to >85 years age group. In general population, women have consistently been shown to have a two-fold chance to develop a depressive disorder compared to men [24]. PD with MDD was seen in greater proportion in female patients which supports the fact that female gender is a risk factor for depression in PD.

Depression in PD is associated with increased disability, worse health-related quality of life and higher caregiver burden [25-26]. PD with MDD patients were transferred more to SNF, INF or another type of facility as compared to PD patients. Also, PD with MDD patients had three times greater chances of disposition to short-term or acute care hospital that may be due to worsened health outcomes. PD with MDD patients had twice the risk of severity of illness and loss of body function compared to PD patients. This likely led to an increased likelihood of dying in PD with MDD patients which indeed was associated with higher in-hospital mortality compared to PD patients.

Psychosis comorbidity was observed in higher proportion of PD with MDD patients followed by other comorbidities like alcohol abuse, drug abuse, and delirium, as compared to PD patients without depression. The presence of psychosis is a strong predictor of MDD in PD patients [27]. So our study supports the observations made earlier. Psychosis and delirium comorbidity was present only in PD with MDD patients and absent in PD patients without depression. Dementia was seen in a greater proportion of PD with MDD patients compared to PD patients without depression. The presence of comorbidities and its treatment procedures had significantly reduced the quality of life for PD patients diagnosed with MDD.

Therapeutic procedures were less commonly performed in PD with MDD patients in comparison to PD patients, whereas diagnostic procedures like CT-scan Head, MRI scan and Diagnostic Spinal Tap, and invasive procedures like Gastrotomy were performed in greater proportion in PD with MDD patients. DBS, which is an important treatment option for PD with dyskinesia and motor complications, was about three times less commonly used in PD with MDD patients as compared to PD patients. The lack of consideration for DBS in PD patients with MDD is concerning as these patients are likely to equally do well in terms of the motor and non-motor symptoms in a prospective study, especially if provided with multidisciplinary care including pre- and post-DBS psychiatric follow-up [28]. Overall, PD with MDD patients had a greater median length of stay in the hospital with higher in-hospital mortality and lower total cost of hospitalization when compared to PD patients.

The biggest strength of our study lies in the national representation of the dataset, with a uniform collection of data, through ICD-9-CM codes over five years. This is the first study, to our knowledge, to report the impact of MDD in PD patients regarding hospital outcomes, 
morbidity, mortality, and utilization of therapeutic procedures. Using the NIS dataset, we got a large sample size as we included 63,912 PD patients and 1445 PD patients with MDD. This dataset is subject to minimal reporting bias, and all information is coded independently of the individual practitioner, making it a more reliable source.

However, our study has several limitations. The NIS is an administrative database and lacks the patient level data needed to make accurate clinical associations. Further, such retrospective studies are always subject to selection bias, which might be accentuated by the moderate sensitivity of diagnostic codes for PD and MDD. We could not account for re-hospitalizations, given the nature of the database, although they add to the total inpatient burden. Despite these potential limitations, the NIS database provides an unparalleled population-based perspective on disease associations with systematic and temporal factors and provides a rationale for further in-depth studies.

\section{Conclusions}

Our study establishes the negative impact of depression in PD in terms of hospitalizationrelated outcomes including the illness severity, comorbid conditions, risk of mortality, utilization of diagnostic and therapeutic procedures, length of stay and disposition as compared to PD alone. Further research to guide the development of clinical care models for targeting identification and treatment of depression in PD is warranted to both reduce mortality and morbidity and improve quality of care in PD with MDD.

\section{Additional Information \\ Disclosures}

Human subjects: All authors have confirmed that this study did not involve human participants or tissue. Animal subjects: All authors have confirmed that this study did not involve animal subjects or tissue. Conflicts of interest: In compliance with the ICMJE uniform disclosure form, all authors declare the following: Payment/services info: All authors have declared that no financial support was received from any organization for the submitted work. Financial relationships: All authors have declared that they have no financial relationships at present or within the previous three years with any organizations that might have an interest in the submitted work. Other relationships: All authors have declared that there are no other relationships or activities that could appear to have influenced the submitted work.

\section{References}

1. Alexander GE: Biology of Parkinson's disease: pathogenesis and pathophysiology of a multisystem neurodegenerative disorder. Dialogues Clin Neurosci. 2004, 6:259-280.

2. Kowal SL, Dall TM, Chakrabarti R, et al.: The current and projected economic burden of Parkinson's disease in the United States. Mov Disord. 2013, 28:311-318. 10.1002/mds.25292

3. Dorsey ER, Constantinescu R, Thompson JP, et al. : Projected number of people with Parkinson disease in the most populous nations, 2005 through 2030. Neurology. 2007, 68:384-386. 10.1212/01.wnl.0000247740.47667.03

4. Statistics on Parkinson's. Parkinson's disease foundation (PDF) . (2017). Accessed: August 10, 2017: http://www.pdf.org/parkinson_statistics.

5. ARRA investments in Parkinson's disease . (2010). Accessed: August 10, 2017: https://report.nih.gov/recovery/investmentreports/ViewARRAInvRpt.aspx?csid=286.

6. Todorova A, Jenner P, Ray Chaudhuri K: Non-motor Parkinson's: integral to motor Parkinson's, yet often neglected. Pract Neurol. 2014, 14:310-322. 10.1136/practneurol-2013000741

7. Bernal-Pacheco O, Limotai N, Go CL, et al.: Nonmotor manifestations in Parkinson disease . Neurologist. 2012, 18:1-16. 10.1097/NRL.0b013e31823d7abb

8. Van der Hoek TC, Bus BA, Matui P, et al.: Prevalence of depression in Parkinson's disease: 
effects of disease stage, motor subtype and gender. J Neurol Sci. 2011, 310:220-224. 10.1016/j.jns.2011.07.007

9. Menon B, Nayar R, Kumar S, et al.: Parkinson's disease, depression, and quality-of-life . Indian J Psychol Med. 2015, 37:144-148. 10.4103/0253-7176.155611

10. Cummings JL, Masterman DL: Depression in patients with Parkinson's disease . Int J Geriatr Psychiatry. 1999, 14:711-718.

11. Leentjens AF: Depression in Parkinson's disease: conceptual issues and clinical challenges . J Geriatr Psychiatry Neurol. 2004, 17:120-126. 10.1177/0891988704267456

12. Schrag A: Quality of life and depression in Parkinson's disease . J Neurol Sci. 2006, 248:151157. 10.1016/j.jns.2006.05.030

13. Reijnders JS, Ehrt U, Weber WE, et al.: A systematic review of prevalence studies of depression in Parkinson's disease. Mov Disord. 2008, 23:183-189. 10.1002/mds.21803

14. McDonald WM, Richard IH, DeLong MR: Prevalence, etiology, and treatment of depression in Parkinson's disease. Biol Psychiatry. 2003, 54:363-375. 10.1016/S0006-3223(03)00530-4

15. Nuti A, Ceravolo R, Piccinni A, et al.: Psychiatric comorbidity in a population of Parkinson's disease patients. Eur J Neurol. 2004, 11:315-320. 10.1111/j.1468-1331.2004.00781.x

16. Martignoni E, Godi L, Citterio A, et al.: Comorbid disorders and hospitalisation in Parkinson's disease: a prospective study. Neurol Sci. 2004, 25:66-71. 10.1007/s10072-004-0232-5

17. Gorell JM, Johnson CC, Rybicki BA: Parkinson's disease and its comorbid disorders: an analysis of Michigan mortality data, 1970 to 1990. Neurology. 1994, 44:1865-1868. 10.1212/WNL.44.10.1865

18. Introduction to the nationwide inpatient sample (NIS). Agency for Healthcare Research and Quality. (2017). Accessed: August 10, 2017: https://www.hcup-us.ahrq.gov/nisoverview.jsp.

19. Clinical classifications software (CCS) for ICD-9-CM . (2017). Accessed: August 10, 2017: https://www.hcup-us.ahrq.gov/toolssoftware/ccs/ccs.jsp.

20. IBM SPSS statistics for Windows. (2017). Accessed: August 11, 2017: https://www.ibm.com/analytics/us/en/technology/spss/.

21. Cummings JL: Depression and Parkinson's disease: a review . Am J Psychiatry. 1992, 149:443454. 10.1176/ajp.149.4.443

22. Hantz P, Caradoc-Davies G, Caradoc-Davies T, et al.: Depression in Parkinson's disease. Am J Psychiatry. 1994, 151:1010-1014. 10.1176/ajp.151.7.1010

23. Schrag A, Hovris A, Morley D, et al.: Young- versus older-onset Parkinson's disease: impact of disease and psychosocial consequences. Mov Disord. 2003, 18:1250-1256. 10.1002/mds.10527

24. Weissman MM, Bland R, Joyce PR, et al.: Sex differences in rates of depression: cross-national perspectives. J Affect Disord. 1993, 29:77-84.

25. Schrag A, Jahanshahi M, Quinn N: What contributes to quality of life in patients with Parkinson's disease?. J Neurol Neurosurg Psychiatry. 2000, 69:308-132. 10.1136/jnnp.69.3.308

26. Schrag A, Hovris A, Morley D, et al.: Caregiver-burden in Parkinson's disease is closely associated with psychiatric symptoms, falls, and disability. Parkinsonism Relat Disord. 2006, 12:35-41. 10.1016/j.parkreldis.2005.06.011

27. Tandberg E, Larsen JP, Aarsland D, et al.: Risk factors for depression in Parkinson disease . Arch Neurol. 1997, 54:625-630. 10.1001/archneur.1997.00550170097020

28. Chopra A, Tye SJ, Lee KH, et al.: Underlying neurobiology and clinical correlates of mania status after subthalamic nucleus deep brain stimulation in Parkinson's disease: a review of the literature. J Neuropsychiatry Clin Neurosci. 2012, 24:102-110.

10.1176/appi.neuropsych.10070109 